J Urol. 2009 November ; 182(5): 2274-2278. doi:10.1016/j.juro.2009.07.024.

\title{
Radical Prostatectomy Findings in Patients in Whom Active Surveillance of Prostate Cancer Fails
}

\author{
Amy S. Duffield, Thomas K. Lee, Hiroshi Miyamoto, H. Ballantine Carter, and Jonathan I. \\ Epstein * \\ Departments of Pathology (ASD, TKL, HM, JIE), Urology (HBC, JIE), and Oncology (JIE), The \\ Johns Hopkins Hospital, Baltimore, Maryland
}

\begin{abstract}
Purpose-Little data are available on radical prostatectomy findings in men who experience disease progression following active surveillance.

Materials and Methods-A total of 470 men in our active surveillance program underwent annual repeat needle biopsies to look for progression defined as any Gleason pattern grade 4/5, more than $50 \%$ cancer on any core or cancer in more than 2 cores. Slides were available for review in 48 of 51 radical prostatectomies with progression.
\end{abstract}

Results-The average time between the first prostate biopsy and radical prostatectomy was 29.5 months (range 13 to 70 ), with $44 \%$ and $75 \%$ of the patients showing progression by the second and third biopsy, respectively. There were 31 (65\%) organ confined cases, of which $25(52 \%)$ were Gleason score 6. Of 48 cases 17 (35\%) had extraprostatic extension, 3 had seminal vesicle/ lymph node involvement and $7(15 \%)$ had positive margins. Mean total tumor volume was 1.3 $\mathrm{cm}^{3}$ (range 0.02 to 10.8 ). Of the 48 tumors 13 (27\%) were potentially clinically insignificant (organ confined, dominant nodule less than $0.5 \mathrm{~cm}^{3}$, no Gleason pattern 4/5) and 19\% (5 of 26) of the radical prostatectomies with a dominant tumor nodule less than $0.5 \mathrm{~cm}^{3}$ demonstrated extraprostatic extension, 4 with Gleason pattern 4 . All 10 tumors with a dominant nodule greater than $1 \mathrm{~cm}^{3}$ were located predominantly anteriorly.

Conclusions-Most progression after active surveillance occurs 1 to 2 years after diagnosis suggesting undersampling of more aggressive tumor rather than progression of indolent tumor. Even with progression most tumors have favorable pathology (27\% potentially insignificant). A small percentage of men have advanced stage disease (pT3b or N1). The anterior region should be sampled in men on active surveillance.

\section{Keywords}

prostatectomy; prostatic neoplasms

With increased use of PSA screening and extended transrectal ultrasound directed prostate biopsies, the number of men diagnosed with prostate cancer has increased significantly.

\footnotetext{
Copyright $(\subseteq 2009$ by American Urological Association

* Correspondence: The Johns Hopkins Hospital, The Weinberg Building, Rm. 2242, 401 N. Broadway St., Baltimore, Maryland 21231 (telephone: 410-955-5043; FAX: 443-287-3818; jepstein@jhmi.edu).
} 
Many of these men are older than 65 years and have low to intermediate risk cancers. ${ }^{1}$ To minimize the overtreatment of prostate cancer older men with small volume, low/ intermediate grade prostate adenocarcinoma on biopsy may be offered the option of active surveillance at our institution.

Scant data are available regarding pathological findings in patients who participate in an active surveillance program and in whom surveillance fails due to disease progression on biopsy. In this study we examined the radical prostatectomy findings in these patients to provide insight into the nature of disease at progression, and to suggest how patients may be better selected and followed in the active surveillance program.

\section{METHODS}

A total of 470 men in our active surveillance program underwent annual repeat needle biopsies to look for progression defined as any Gleason pattern grade $4 / 5$, more than $50 \%$ cancer on any core or cancer in more than 2 cores. A detailed description of our selection and followup plan for this program is available in prior publications. ${ }^{2}$ Slides were available for review in 48 of 51 RPs with progression. All RP specimens were fixed in formalin, sliced at $3 \mathrm{~mm}$ intervals and the prostate was embedded in entirety. Tumor volume was determined using the grid method. ${ }^{3}$

\section{RESULTS}

Mean patient age at presentation was 62 years (range 52 to 70). Average number of biopsies performed during active surveillance was 3.1 (range 2 to 8 ). Average time between first prostate biopsy and RP was 29.5 months (range 13 to 70 ) with $44 \%$ of patients experiencing progression on the second biopsy and 75\% (36 of 48) by the third biopsy. There were 33 men who had only 1 of the adverse pathological criteria on final biopsy and the remaining 15 had 2 or more adverse pathological findings (table 1). Average PSA at diagnosis was $6.22 \mathrm{ng} / \mathrm{ml}$ (range 2.1 to 21.2) and average PSA at RP was $6.15 \mathrm{ng} / \mathrm{ml}$ (range 2.7 to 14.6) in the 31 patients for whom these data were available. A total of 22 patients had an increase in PSA, averaging an increase of $1.82 \mathrm{ng} / \mathrm{ml}$ (range 0.1 to 8.5 ), and 9 had a decrease in PSA, averaging a decrease of $4.19 \mathrm{ng} / \mathrm{ml}$ (range 0.4 to 12.7 ).

Radical prostatectomy findings are summarized in table 2 . There was no significant difference in RP tumor volume whether the needle biopsies contained only 1 needle biopsy criterion for progression or 2 to 3 criteria. Of the 48 tumors 13 (27\%) were potentially clinically insignificant (organ confined, dominant tumor less than $0.5 \mathrm{~cm}^{3}$, no Gleason pattern 4 or 5). Of the RPs with a dominant tumor nodule less than $0.5 \mathrm{~cm}^{3}, 19 \%$ (5 of 26) demonstrated EPE and 4 had GP 4. Altogether 34 of 48 (71\%) RPs showed at least 1 of the criteria of EPE, GP 4 or total tumor volume greater than $1 \mathrm{~cm}^{3}$. A small percentage had advanced stage disease (pT3b or N1).

All 10 tumors with a dominant nodule greater than $1 \mathrm{~cm}^{3}$ were located predominantly in the anterior aspect of the prostate with 9 of 10 involving the transition zone, although 3 of these tumor nodules also focally extended posteriorly (table 3 ). In contrast there was no grade or 
stage anterior predilection in this group of men, and 14 of 29 (48\%) GP 4/5 and 8 of 17

(47\%) pT3 tumors were predominantly localized anteriorly.

In all RPs at least 1 positive biopsy core corresponded to the region of the dominant tumor nodule, suggesting that the dominant tumor nodules were sampled on biopsy in all cases. The number of biopsies that a patient had was not significantly associated with tumor volume. Of 48 cases 10 had perineural invasion on needle biopsy with 4 of $10(40 \%)$ having EPE ( 3 focal, 1 nonfocal). The percentage of EPE in the remaining 38 RPs that did not show perineural invasion on biopsy was similar (13 of $38,38 \%$ ).

\section{DISCUSSION}

With the advent of PSA screening and transrectal ultrasound directed prostate biopsies the number of men diagnosed with prostate cancer has increased significantly. Many of these men are older than 65 years and have low to intermediate risk cancers, ${ }^{1}$ and before PSA screening a significant number of these cancers would have remained undetected. ${ }^{4}$ Most men who are diagnosed with low to intermediate risk cancers undergo treatments that may be associated with significant morbidity but the majority of the older men who have active treatment for screen detected prostate cancer do not live longer due to the medical intervention for prostate cancer. ${ }^{1,2}$

To minimize the overtreatment of prostate cancer older men with small volume, low/ intermediate grade prostate adenocarcinoma on biopsy may be offered the option of active surveillance at our institution. Overall approximately $1 \%$ to $2 \%$ of patients who are seen at our institution for newly diagnosed prostate cancer choose the active surveillance program. ${ }^{2}$ Ideally these patients are at least 65 years old. However, some younger patients enter into this program due to patient preference or comorbidities that preclude active treatment. To qualify for the active surveillance program at our institution the tumor must be nonpalpable and PSAD must be less than $0.15 \mathrm{ng} / \mathrm{ml} / \mathrm{cm}^{3}$. The prostate biopsy must have no Gleason pattern grade 4 or 5 , and 2 or fewer cores may be involved by cancer. None of these cores may have more than $50 \%$ involvement by carcinoma. ${ }^{5}$ Although the PSAD value is integral for patient inclusion in our active surveillance program, we noted that the overlap in PSAD values and other PSA derived measurements (ie PSA velocity, free/total PSA) among those with and without progression precludes their use for an individual in predicting progression. ${ }^{2}$ Rather we solely rely on subsequent biopsy pathology to determine progression.

Our early finding in the active surveillance program was that in the majority of cases more extensive disease was found on surveillance biopsies in the first 2 years of followup as also seen in the current study. We recognized that our initial followup protocol, in which inclusion biopsies often only sampled the prostate in a sextant fashion, was inadequate and often under sampled the tumor. Recognizing the limitations of our criteria to correctly identify those men with small volume cancer and data indicating the importance of posterolateral biopsies in excluding significant cancers, we now recommend that all men who are considering expectant management undergo repeat wide area sampling of the prostate using ultrasound needle directed biopsies to decrease the odds that the initial biopsy 
underestimated the extent of disease. ${ }^{6}$ Thus, we are sampling the midsagittal peripheral zone at the base, mid and apex as well as the far lateral peripheral zones of the prostate. ${ }^{7}$

The cohort of men in the active surveillance program has been followed since the program inception in 1995, and as of November 7, 2006 approximately 60\% were still undergoing active surveillance, $25 \%$ had undergone curative interventions, approximately $10 \%$ had withdrawn from the program and $5 \%$ were lost to followup or died of other causes. ${ }^{2}$ Prior review of the pathological data demonstrated that men who were in the active surveillance program had similar rates of incurable cancer compared to those who qualified for the program but had immediate surgery, and our findings at this later date are commensurate with the early findings. ${ }^{2,8}$

While these earlier studies had considered the TNM stage of the tumor, tumor volume and location were not determined. In this study we extended prior work to investigate the total tumor volume as well as the volume and location of the dominant nodule. Some of the smaller tumors identified in the current study were fairly aggressive based on grade or stage. Due to their small size these would have been difficult to detect by PSA and were fortunately detected by biopsy. Not all groups with active surveillance programs perform annual biopsies as is done at our institution, with some simply following patients by PSA kinetics after the first 2 biopsies and others advocating biopsies at longer intervals. ${ }^{9}$ None of the patients in this study had treatment failure with active surveillance due to PSA. PSA values are imperfect markers of cancer progression and their use in active surveillance programs is still being refined. ${ }^{10}$ The serial biopsies we performed did ultimately identify several patients who did not have alarming PSA values but had disease that was likely to progress.

A potential criticism of serial biopsies based on the current study is that it is more likely to identify some men as having experienced treatment failure on active surveillance despite having low risk disease. Approximately a quarter of the patients with progression had potentially clinically insignificant prostate cancer at prostatectomy. However, when following men with prostate cancer we would rather err on the side of overtreating some in our active surveillance program as opposed to not actively treating some with significant cancers. Repeat biopsy is the best means to find those patients with small foci of high grade disease and provide them with the appropriate definitive treatment.

One of the most consistent findings in the current study was the association of a larger dominant tumor nodule with anterior/transition zone tumor. The majority of prostate cancers arise in the posterior aspect of the prostate. In routine practice transition zone biopsies are rarely positive and, thus, have limited usefulness. ${ }^{11}$ However, in our active surveil-lance cohort of patients the large tumors that were missed by the biopsy protocol had a marked tendency to involve the transition zone. Tumors located in the transition zone are considered less aggressive, and are associated with a lower stage, less EPE and less seminal vesicle involvement than other prostate carcinomas. ${ }^{12-14}$ However, there is a subset of carcinomas arising in the transition zone that is characterized by adverse pathological features and aggressive behavior. ${ }^{15}$ While many anterior dominant cancers also have concurrent additional tumor foci in the posterior prostate, some of these anterior dominant tumors are 
not detected through standard transrectal biopsies, or the tumor that is detected on biopsy is not extensive enough to meet exclusion criteria for active surveillance despite the presence of a substantial tumor in the prostate. ${ }^{15,16}$

Tumors in the anterior aspect of the prostate are difficult to assess clinically because they are difficult to image, palpate on digital rectal examination and sample on transrectal biopsy. ${ }^{13}$ Some groups have used a combined transrectal and transperineal approach which more effectively samples the transition zone. This method increases the cancer detection rate but is associated with increased morbidity as well as increased pain and discomfort. ${ }^{17}$

Alternatively transrectal anterior directed biopsies may be performed, typically as the last set of biopsies performed in a given patient. It has been shown that anterior tumors often require more sets of biopsies for detection and the amount of tumor in the biopsies is less than the amount of tumor from equivalent size posterior tumors. ${ }^{18}$ Given that percentage involvement of a biopsy core by tumor is an inclusion criterion for active surveillance, it is crucial that the biopsy be able to determine the amount of tumor in the anterior/transition zone with reasonable accuracy. Based on the current study data we have modified our repeat biopsy protocol to include anterior/ transition zone sampling.

We recognize that active surveillance, like prostate cancer screening, is controversial. A criticism of active surveillance is that the window of opportunity for cure will be lost in some men. However, there is high level evidence from a randomized trial of surgery vs watchful waiting in men with nonscreen detected prostate cancer that the benefits of surgery are restricted to men younger than 65 years. ${ }^{19}$ Thus, the evidence would suggest that surveillance for carefully selected, older men with screen detected prostate cancer is safe. Our policy is to recommend curative intervention for healthy younger men with low risk prostate cancers to avoid harm. For those younger men who insist on active surveillance for personal reasons we believe that a policy of careful monitoring with established biopsy criteria for intervention is a safer approach than no monitoring at all.

\section{CONCLUSIONS}

Even with progression on biopsy following active surveillance most tumors are organ confined, GS 6 with negative margins and relatively low tumor volumes, and 27\% of the tumors are potentially insignificant cancers. However, despite minimal tumor on biopsy and yearly biopsies a small percentage of men have advanced stage disease (pT3b or N1). Men considering active surveillance should be counseled on the risk of progression and the likelihood of cure if progression occurs. In addition to an initial extended biopsy to minimize the risk of undersampling cancer at enrollment in an active surveillance program, repeat biopsies should sample the anterior aspect of the prostate because many of the larger tumors that evade detection on biopsy are located in the anterior prostate, particularly the transition zone. With careful selection and improvements in active surveillance protocol, patients with low risk prostate cancer can be monitored in a more effective manner while avoiding the morbidity associated with cancer treatments. 


\section{Abbreviations and Acronyms}
EPE extraprostatic extension
GP Gleason pattern
GS Gleason score
PSA prostate specific antigen
PSAD prostate specific antigen density
RP radical prostatectomy

\section{REFERENCES}

1. Cooperberg MR, Moul JW, Carroll PR. The changing face of prostate cancer. J Clin Oncol. 2005; 23:8146. [PubMed: 16278465]

2. Carter HB, Kettermann A, Warlick C, et al. Expectant management of prostate cancer with curative intent: an update of the Johns Hopkins experience. J Urol. 2007; 178:2359. [PubMed: 17936806]

3. Yoon GS, Wang W, Osunkoya AO, et al. Residual tumor potentially left behind after local ablation therapy in prostate adenocarcinoma. J Urol. 2008; 179:2203. [PubMed: 18423736]

4. Draisma G, Boer R, Otto SJ, et al. Lead times and overdetection due to prostate specific antigen screening: estimates from the European Randomized Study of Screening for Prostate Cancer. J Natl Cancer Inst. 2003; 95:868. [PubMed: 12813170]

5. Epstein JI, Walsh PC, Carmichael M, et al. Pathological and clinical findings to predict tumor extent of nonpalpable (stage T1c) prostate cancer. JAMA. 1994; 271:368. [PubMed: 7506797]

6. Epstein JI, Walsh PC, Carter HB. Importance of posterolateral needle biopsies in the detection of prostate cancer. Urology. 2001; 57:1112. [PubMed: 11377320]

7. Carter HB, Walsh PC, Landis P, et al. Expectant management of nonpalpable prostate cancer with curative intent: preliminary results. J Urol. 2002; 167:1231. [PubMed: 11832703]

8. Warlick C, Trock BJ, Landis P, et al. Pathologic outcomes are similar for men in an expectant management program undergoing delayed surgical intervention compared to those undergoing immediate intervention. J Natl Cancer Inst. 2006; 98:355. [PubMed: 16507832]

9. Klotz L. Active surveillance for prostate cancer: trials and tribulations. World J Urol. 2008; 26:437. [PubMed: 18813934]

10. van den Bergh RC, Roemeling S, Roobol MJ, et al. Prostate-specific antigen kinetics in clinical decision-making during active surveillance for early prostate cancer-a review. Eur Urol. 2008; 54:505. [PubMed: 18585845]

11. Epstein JI, Walsh PC, Sauvageot J, et al. Use of repeat sextant and transition zone biopsies for assessing extent of prostate cancer. J Urol. 1997; 158:886.

12. Magheli A, Rais-Bahrami S, Peck HJ, et al. Importance of tumor location in patients with high preoperative prostate specific antigen levels (greater than $20 \mathrm{ng} / \mathrm{ml}$ ) treated with radical prostatectomy. J Urol. 2007; 178:1311. [PubMed: 17698095]

13. Al-Ahmadie HA, Tickoo SK, Olgac S, et al. Anterior-predominant prostatic tumors: zone of origin and pathologic outcomes at radical prostatectomy. Am J Surg Pathol. 2008; 32:229. [PubMed: 18223325]

14. Bouyé S, Potiron E, Puech P, et al. Transition zone and anterior stromal prostate cancers: zone of origin and intraprostatic patterns of spread at histopathology. Prostate. 2009; 69:105. [PubMed: 18850578]

15. Shannon BA, McNeal JE, Cohen RJ. Transition zone carcinoma of the prostate gland: a common indolent tumour type that occasionally manifests aggressive behaviour. Pathology. 2003; 35:467. [PubMed: 14660095] 
16. Noguchi M, Stamey TA, McNeal JE, et al. Prognostic factors for multifocal prostate cancer in radical prostatectomy specimens: lack of significance of secondary cancers. J Urol. 2003; 170:459. [PubMed: 12853799]

17. Watanabe M, Hayashi T, Tsushima T, et al. Extensive biopsy using a combined transperineal and transrectal approach to improve prostate cancer detection. Int J Urol. 2005; 12:959. [PubMed: 16351651]

18. Bott SR, Young MP, Kellett MJ, et al. Anterior prostate cancer: is it more difficult to diagnose? BJU Int. 2002; 89:886. [PubMed: 12010233]

19. Bill-Axelson A, Holmberg L, Filén F, et al. Radical prostatectomy versus watchful waiting in localized prostate cancer: the Scandinavian prostate cancer group-4 randomized trial. J Natl Cancer Inst. 2008; 100:1144. [PubMed: 18695132] 


\section{Table 1}

\begin{tabular}{|c|c|c|}
\hline \multicolumn{3}{|c|}{ Needle biopsy findings at progression } \\
\hline \multirow{6}{*}{ 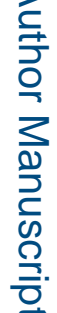 } & & No./Total No. $(\%)$ \\
\hline & Multiple core (more than 2) involvement & $14 / 48(29)$ \\
\hline & GP $4 / 5$ & $13 / 48(27)$ \\
\hline & More than $50 \%$ involvement of core & $6 / 48(13)$ \\
\hline & Multiple cores + more than $50 \%$ core involvement & $6 / 48(13)$ \\
\hline & GP $4 / 5+$ more than $50 \%$ involvement of core & $4 / 48(8)$ \\
\hline & GP $4 / 5+$ multiple cores & $3 / 48(6)$ \\
\hline & All 3 adverse criteria & $2 / 48(4)$ \\
\hline & Total & $48 / 48(100)$ \\
\hline
\end{tabular}




\section{Table 2}

\section{Radical prostatectomy findings}

\begin{tabular}{lr}
\hline Mean gm RP (range) & $75.5(34.3-152.8)$ \\
No./total No. GS (\%): & $19 / 48(39.6)$ \\
6 & $6 / 48(12.5)$ \\
6 With tertiary pattern 4 & $15 / 48(31.3)$ \\
$3+4=7$ & $6 / 48(12.5)$ \\
$4+3=7$ & $2 / 48(4.2)$ \\
Greater than 7 & $31 / 48(65)$ \\
No./total No. organ confined $(\%)$ & \\
No./total No. EPE (\%): & $7 / 48(14.6)$ \\
Focal & $10 / 48(20.8)$ \\
Nonfocal & $1 / 48(2.1)$ \\
No./total No. seminal vesicle involvement $(\%)$ & $2 / 48(4.2)$ \\
No./total No. lymph node involvement $(\%)$ & $7 / 48(15)$ \\
No./total No. pos margins $(\%)$ & $1.3(0.02-10.8)$ \\
Mean cm ${ }^{3}$ total tumor vol (range) & $33 / 48(68)$ \\
No./total No. total tumor vol less than $1 \mathrm{~cm}^{3}(\%)$ & $1.03(0.01-10.57)$ \\
Mean cm ${ }^{3}$ dominant tumor nodule vol (range) & $10 / 48(20.8)$ \\
No./total No. dominant tumor nodule vol greater than $1 \mathrm{~cm}^{3}(\%)$ & \\
\hline
\end{tabular}




\section{Table 3}

Radical prostatectomy predominant tumor location

\begin{tabular}{lr}
\hline & No./Total No. (\%) \\
\hline Dominant nodule: & \\
Anterior & $20 / 48(41.7)$ \\
Posterior/posterolat & $23 / 48(47.9)$ \\
Lateral & $4 / 48(8.3)$ \\
Scattered foci & $1 / 48(2.1)$ \\
Dominant nodule greater than $1 \mathrm{~cm}^{3}:$ & \\
Anterior & $10 / 10(100)$ \\
Dominant nodule less than $1 \mathrm{~cm}^{3}:$ & \\
Posterior/posterolat & $23 / 38(60.5)$ \\
\hline
\end{tabular}

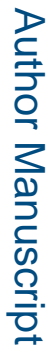

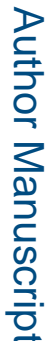

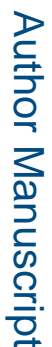

J Urol. Author manuscript; available in PMC 2015 April 08. 